Dr. Edward J. Bernstein, Kalamazoo, Mich.: Will the author please explain what he means by hypertension, and by high blood pressure? Many of us are under the impression that hypertension means high blood pressure. I should like to ask how the author arrives at a differential diagnosis in nephritic troubles when there are neither casts nor albumin in the urine.

Dr. G. E. DE Schwernitz, Philadelphia: It is worth while to emphasize that the so-called diagnostic or characteristic eyeground of nephritis is not really a characteristic eyeground. I believe that there is a period in the progress of nephritis which we may call what the French speak of as the incidence of nephritis. It consists in a curious change in the nerve head. It is important also to emphasize the fact that we are not yet prepared to speak of the diagnostic eyeground in relation to the clinical symptomatology. Because a man has some retinal changes he must not be condemned to death in one year or eighteen months. He may die within such period after changes have been found with the ophthalmoscope, but not necessarily so. Under modern treatment prolongation of life to many months and even years is possible.

Dr. S. D. Risley, Philadelphia: We should speak of these retinal changes as being a toxic retinitis instead of a nephritic retinitis, because these same changes are present in kiflney disease and other toxic affections. They may be associated with cardiovascular disease which is associated with kidney affections and with other nutritional diseases. It is a very rare case of glycosuria in which we do not find this toxic retinitis present.

Dr. Edward Jackson, Denver: Conservatism in prognosis is a good thing to cultivate. A case in point is that of a man with very marked instability of the nervous system, vascular retinal changes, typical, as we supposed, of high blood pressure. 'He took up golf and has devoted two or three afternoons every week to it, where he had done nothing of the kind before. He has been under my observation some six ycars and prognosis from a retinal examination today would be entirely favcrable. Another case typical of vascular change happened in a woman of 60 . That patient lived without any particular change of habits for over six years, with gradual deterioration of vision but with not much other change that could be connected with the retinal condition. $A$ third case is that of a medical friend who accidentally, in good health, discovered that he had a systolic blood pressure of $235 \mathrm{~mm}$. He has been able to reduce it to 180 . He is comparatively young-under 40 . He has changed his method of iife largely, but continues in good health, and with very little change in his retinal vessels.

Dr. Walter R. PArker, Detroit: One interesting fact elicited by this work was the relation of the swelling of the nerve in every case to, or its association with, faulty eliminaiion. No matter where we classify it, we have faulty elimination when there is swelling in the retina and in the nerve head.

Dr. George Slocum, Ann Arbor, Mich.: We classify diseases by the grouping of symptoms as based on clinical observation. A patient with chronic interstitial nephritis will live to be old. Patients with chronic nephritis with large white kidney seldom live to be old. Of course no case of chronic nephritis exists a long time without vascular changes. We must always await the laboratory diagnosis in the great class of cases. No internist makes a diagnosis of nephritis, or even of the type of nephritis, until he has made a careful study of his laboratory findings; not from cne examination, but a series of examinations

As to what is hypertension as contrasted with high blood pressure, if we have high blood pressure existing by itself, with no other discoverable predominating feature, I understand it has been the custom in the clinic to diagnose hypertuision; otherwise the case was diagnosed according to the features found by laboratory study. As to how to make a differential diagnosis in the various forms of nephritis when neither casts nor albumin are present, I am unable to answer that question. Inasmuch as these cases were diagnosed in the clinic of internal medicine, I did not think it necessary to go into the diagnosis. "One point you will note, that the only ophthalmoscopic feature that appeared in the nephritis cases was radiating macular changes. In our clinic a number of years ago we had a case of radiating macular figure as typical as could be, and at last report the patient was alive and well. There were no laboratory findings; at least, no nephritis was present in this case, but it was striking that the radiating macular figure was present in one eye only. Other cases of similar type have appeared, but as to their subsequent histories I am not informed.

\section{PARENCHYMATOUS DISEASE OF THE LIVER A CAUSE FOR RISE IN PORTAL BLOOD PRESSURE}

\section{F. HOOVER, M.D. CLEVELAND}

In attempting an explanation for ascites in hepatic cirrhosis, we must consider the blood pressure and minute volume of flow in the aorta and cava. If the pressure and flow of blood in the aorta and cava are unchanged, then obviously the only hydraulic considerations are those which may affect blood pressure within the portal vein.

There is an abundance of evidence from pathologic sources to prove that ascites may be caused by stenosis of the portal trunk from pylephlebitis. In many instances of hepatic syphilis, ascites is due solely to this source. If the portal pressure is gradually raised during a long period of time, anastomoses form which may be sufficient to keep the portal pressure sufficiently low to prevent ascitic accumulations. But these factors alone are not sufficient to explain why some cirrhoses of the liver are attended with ascites and others are not, when the entire group of ascitic cases are uncomplicated with disease of the radicles, trunk or large branches of the portal vein. Pathologic histology fails to explain why only one of two cirrhotic livers may produce ascites. So far as gross appearances and histologic findings go, we lack an explanation for ascites in the amount and distribution of fibrous tissue throughout the liver.

From our clinical experiences in the study of pylephlebitis and portal anastomoses with tributaries to the cava, it seems clear that a rise in pressure in the portal vein must precede ascites. Here, however, we encounter experiences which are very disconcerting to this view of ascites if we see in a rise of portal vein pressure only an expression of obstruction to the portal flow from fibrous tissue formation. Every clinician sees patients with typical alcoholic cirrhosis who will have ascites which accumulates very rapidly. The ascitic fluid is drawn off one or more times, and, in spite of the fact that there is no demonstrable change in the hepatic signs, the patient will live for some years without a return of ascites.

There is then some temporary alteration in the liver which causes ascites. Thus far I have never read of any direct evidence which goes to prove that these transient periods of ascites are dependent on a coincidentally transient rise in portal pressure. The rise in portal pressure with its consequent ascites cannot bear any relation to fibrous tissue formation. The cause must lie in some other source of resistance to the portal flow of blood.

The portal cirrhosis in cases of this kind proceeds uninterruptedly, and the source for increased portal pressure and ascites must obviously be sought else- 
where than in the cirrhotic process. F. C. Herrick ${ }^{1}$ made a comparative study of the minute-volume flow through the portal vein and hepatic artery in normal livers and in the livers of interstitial hepatitis removed at necropsy. Herrick found that interstitial hepatitis did not cause any obstruction to the flow of normal salt solution or defibrinated blood. Perfusion of the portal vein of cirrhotic livers gave as large a minutevolume flow as that procured from perfusion of normal livers. He did not find, however, that the anatomic interrelation between portal vein branches and hepatic arterial branches was very different in cirrhotic livers from the interrelation between the vein and artery of normal livers. In cirrhotic livers the portal pressure rose much higher under the influence of elevated pressure in the hepatic artery than it did in normal livers.

Herrick's experiments show that there is a different relation between the hepatic arterial branches of the portal vein from that which exists normally. But what vitiates the significance of his results is the fact that the minute-volume flow through the hepatic artery in Herrick's dead livers was in all cases greater than the minute-volume flow through the portal vein. In life the reverse is true, the minute-volume flow through the portal vein being twice as large as the arterial minute-volume. This disparity between the results of postmortem perfusion and the measurements of minute-volume blood flow in vivo can be accounted for by a loss of vasomotor arterial tone in the dead livers. In the portal vein, however, the vasomotor supply to the intrahepatic branches is very feeble. Although Herrick does not offer a satisfactory explanation for the portal vein pressure in hepatic cirrhosis, he does show that the interstitial fibrous tissue of a portal cirrhosis will not account for an elevation in pressure within the portal vein.

The state of the liver cells and edema of the liver have been neglected in considering the causes for rise of the portal pressure in hepatic cirrhosis.

The clinical histories of hepatic cirrhosis contain periods of subacute exacerbations of symptoms from the biliary and portal systems, for example, transient cholemia with transient ascites. During these subacute exacerbations the liver increases much in size and becomes tender to pressure. As cholemia and ascites subside, the liver grows smaller and is no longer sensitive to pressure. There has always been a lack in the chain of evidence, however, to prove that these transient ascites are really associated with an elevation of pressure in the portal vein.

We recently had in our wards at Lakeside Hospital a patient who supplies the wanting link in the chain of evidence to prove that these attacks of subacute parenchymatous hepatitis are actually accompanied by a rise in portal blood pressure.

\section{REPORT OF CASE}

CASE 1.-F. K., man, aged 35, had used alcoholics in excess during his aduit life. Three weeks before he was admitted to the hospital he noticed that his feet, legs and abdomen were swollen. He stopped work and quit drinking alcoholics for a few days and the swelling disappcared, whereupon he resumed work and also the use of beer and whisky. Ten days before entering the hospital the swelling in his lower exiremities and abdomen returned, and he became jaundiced for the first time.

When admitted to the hospital, the patient was jaundiced, there was edema of the feet, legs and scrotum, and a great

i. Herrick, F. C.: Jour. Exper. Med., 1907, ix, 93. amount of ascitic fluid behind which a large hardened liver could be palpated a hand's breadth below the costal margin. There was no free fluid in the pleural or pericardial sacs, and there was not the slightest evidence to indicate any disturbance in the cardiovascular or pulmonary systems. The right auricle and left ventricle were not enlarged. The blood pressure was 130 systolic and 90 diastolic. The temperature was 102 . The urine contained bile pigment and bile salts and a slight trace of albumin. In the sedinent were a few hyaline and granular casts. There was a well developed caput medusae in the supra-umbilical region. Midway between the umbilicus and the ensiform cartilage to the left of the median line was a veritable bulbus venosus over wh:ch there was a palpable thrill and a loud venous hum.

Two days after entering the hospital the abdomen was tapped, and 8,000 c.c. of a clear yellow serum rcmoved. The specific gravity was 1.005 , albumin $4.5 \mathrm{gm}$. per liter, and there were 180 cells per cubic millimeter.

The cells were largely mononuclear. The blood was drawn from a vein into a 1 per cent. potassium oxalate solution and centrifuged. The plasma was deeply tinged with bile pigment, and when diaiyzed from a collodion sack against equal parts of alcohol and water, the dialysate contained both bile pigment and bile salts. The plasma gave no reaction to the paraldehyd test for urobilin, but the urine contained an abundance of urobilin as well as both bile pigment and bile salts. The stool was dark colored and gave a reaction to urobilin which was still visible spectroscopically when the daily stool was diluted to 1 liter with water and a part of this mixture was again diluted $1: 2,500$ with water. The concentration of bile pigment in the plasma was much greater than in the ascitic fluid. The blue-green band between the coagulum and plasma which occurs when nitric acid is poured beneath the plasma was very marked, but when the nitric acid contact test was made on the ascitic fluid the color band was very faint. The patient's fever rose as high as 102 November 11, the day of admission. On November 12 and 13, the tem. perature rose as high as 103 and 100 , respectively. The blood contained $5,180,000$ red blood corpuscles, 6,400 white blood corpuscles, and hemoglobin 85 per cent. (Tallqvist).

Between November 13 and 22 the temperature declined to normal and remained normal for the remaining four days that the patient was in the hospital.

Several notable things occurred during the interval between November 13 and 22. The venous hum over the caput medusae was unaffected by the removal of the ascitic fluid. It was quite as loud after the intra-abdominal pressure was relieved as it was before the tapping; but by November 22, nine days after the tapping, the murmur and thrill over the caput medusae were gone, although the venous anastomoses were as plainly visible as before. In the interval of nine days above referred to, the ascites entirely disappeared. The liver grew much smaller. The liver border ascended three finger breadths so that the lower sharp edge was palpable only about an inch below the costal margin at the nipple line. The left and right lobes of the liver diminished proportionately in size, and all liver tenderness was gone. After the third day, there were no longer any casts or albumin in the urine. All these changes occurred independently of any alteration in the size of the heart or change in blood pressure in the aortic system. November 17 the urine still contained bile salts and bile pigment, but the urobilin content of the stool was enormously increased, as the retention of bile in the blood had ceased and a marked hypercholia was instituted as the liver began to eliminate the large amount of bile and bile salts which had accumulated in the blood. The patient was greatly improved in all symptoms, and the stool showed the test for urobilin in dilutions of $1: 44,600$, whereas on entrance the test showed only in dilutions as high as $1: 2,500$. The patient was evidently recovering from his acute hepatitis, and the iiver was recovering its biliary function. November 22 , nine days after admission, the urine no longer contained bile salts or bile pigment, nor were there any biliary elements found in the urine during the remainder of his stay in the hospital, which terminated November 26. 
November 26, the murmur and thrill over the caput medusae were gone, and there was no free fluid in the abciomen or edema elsewhere in the body. The liver was much diminished in size. The jaundice was much less marked, there was no choluria, and the oxalate plasma showed a grcat diminution in the cholemia. There was still sufficient bile pigment to give the nitric acid contact test, and the dialysate from the plasma gave Hammarsten's test for bile pigment; but Pettenkofer's test for bile salts in the plasma dialysate was negative.

\section{COMMENT}

In the size of the liver, the improvement in the cholemia and recovery from choluria, and the great intestinal hypercholia and recovery from ascites, we have the clinical picture of recovery from a subacute hepatitis. Coincidentally with this improvement, the murmur and thrill in the caput medusae disappeared. The murmur in the caput medusae is due to two factors, namely, the conformation of the venous channel and the velocity of the blood stream. The character of the blood was unchanged, and the conformation of the veins remained the same; the only change was in the velocity of the blood in the anastomotic veins. The velocity of the blood in this location was an expression of the disparity between the pressure in the portal vein and in the superior vena cava. It indicated the transit of blood from a field of high pressure to a field of low pressure. When the portal pressure diminished with recovery from subacute hepatitis, the disparity between the portal vein and cava pressures grew less and the velocity of the venous current diminished sufficiently to make the thrill and murmur disappear. The effect of posture on the venous hum at the bulbus venosus in the right supraclavicular region jllustrates with what ielicacy the critical point of velocity in the venous stream is determined at which a murmur will develop.

If a patient who has a venous hum of anemia sits upright, the hum will be loud at the bulbus venosus. If the patient is slowly let down from the upright toward the recumbent position, an angle of inclination will be found at which the murmur ceases. If the patient sits upright, the murmur is very loud in a given instance. When the patient is let down toward the horizontal position, the murmur will still be loud when the trunk forms an angle of 35 degrees with the bed. If he is let down further so that the trunk forms an angle of 30 degrees with the bed, the murmur is no longer audible. The angle at which the murmur will disappear varies in different cases, but in all cases the critical point at which the murmur disappears is sharply defined. The transition from a loud murmar to silence over the bulbus venosus occurs within a change in inclination of the body of a very few degrees. In nearly all cases of anemia, the nurmur ceases before the patient reaches the horizontal position. I have found only two exceptions to this rule, and they were both in patients who had anemia due to lead poisoning and who, in addition to their anemia, had an elevation of systolic and diastolic blood pressures, due to hypertonus of the splanchnic arteries. In both these cases the murmur was still loud when the patients were in the horizontal position. The murmur did not disappear until the foot of the bed was elevated about 2 feet from the floor. This of course involves the question of minute-volume flow through the encephalon attending splanchnic arterial hypertonus. However this may be, the observations prove that employment of gravity shows how delicately the velocity of the venous current determines the appearance or disappearance of the venous hum.

To return to our cirrhotic liver patients: In the caput medusae there was a loud venous hum and palpable thrill before and after the ascitic fluid was withdrawn; without any alteration in the hydraulics of the systemic blood flow, the murmur disappeared coincidentaily with recovery from the subacute parenchymatous hepatitis, and furthermore, after recovery from all the signs of acute hepatitis, there was no return of the ascites. It seems, therefore, that we have very good proof that subacute parenchymatous hepatitis in the course of chronic interstitial alcoholic cirrhosis may cause sufficient elevation in portal blood pressure to produce a large ascitic accumulation which does not reaccumulate when the portal pressure is lowered.

In Case 1, the liver was enlarged and sensitive. The tenderness on pressure was due to the bursting tension of Glisson's capsule and not to perihepatitis. The liver was equally sensitive over the entire breadth of the right and left lobes, and there was no perihepatic friction palpable or audible. The ascitic fluid had all the characteristics of a transudate.

In Case 2, there was an acute afebrile disease of the liver accompanied by ascites and intense jaundice and attended with grave signs of intoxication ascribable to impairment of hepatic function; but, during the height of the disease, the consistency of the liver was diminished and there is evidence to show that the tension of Glisson's capsule was less than normal during the period in which ascites accumulated.

\section{REPORT OF CASE}

CASE 2.-A man, aged 21, admitted to Lakeside Hospital Oct. 12, 1911, and discharged Dec. 19, 1911, had been observed to have jaundice two weeks prior to admission. The patient was confined to bed only one week before he was brought to the hospital. On admission there was intense jaundice, an abundance of bile in the urine, and the stools showed no indication of a want of bile. The pulse was 52 . The heart was not enlarged; the systolic arterial pressure was 130 , and the diastolic pressure 90 . The temperature was 98 . The liver was enlarged and slightly sensitive to pressure. The hepatic area of dulness in the nipple line extended from the fifth rib to $3 \mathrm{~cm}$. below the costal margin. The hepatic edge was easily palpated, and the liver was sensitive to pressure. The spleen had an enlarged area of percussion dulness, but did not reach quite to the costal margin. The abdominal wall was rather firm, and did not admit palpation of the splenic border. The subsequent clinical course shows further progress in hepatic disease to the point where marked hypocholia, ascites, hemorrhages, acidosis and violent convulsions all developed. During this period there were no evidences of cardiac or renal impairment. Then followed signs of recovery with a transient increase of cholemia, which had subsided during the hypocholic period.

During the period of maximum severity of the disease, when hypocholia, hemorrhages, ascites and convulsions all developed, the liver diminished in consistency, and had a lowered tension of Glisson's capsule.

During the subsequent period of recovery, the liver regained its original size and consistency. In this case of acute hepatitis, ascites developed during a period of maximum hepatitis when the volume of the liver was apparently diminished and tension on Glisson's capsule was lessened, whereas in Case 1, ascites developed during a period of subacute hepatitis during which period the liver was enlarged and the tension on Glisson's capsule was evidently much increased:

The following are extracts from the bedside notes:

October 14: Test carbohydrate meal removed one hour after eating showed free hydrochloric acid 8 , total acidity 38 , benzidin reaction negative, lactic acid negative. 
October 15: Stool light brown, no parasites or parasite eggs in the stool. No fat or blood cells. Urobilin present in abundance.

October 17: Vomited 400 c.c. after a meal of toast, oatmeal and milk. Acid to litmus. Hydrochloric acid deficit 24. Total acidity 59 .

October 19: Although there is no abdominal distention from gas or meteorism, the hepatic area of dulness is much diminished and the liver is no longer palpable. The epigastrium is retracted. In the nipple line, there is hepatic dulness only from the sixth to the eighth ribs. From this point, the distance from the eighth rib to the costal border is $7 \mathrm{~cm}$. In following the seventh interspace from the costal margin there is no hepatic dulness encountered until the anterior axillary line is reached. The liver seems to have fallen in on itself. It has receded from the boundarics of the abdominal wall and is no longer palpable.

Vomited coffee ground material. Free hydrochloric acid 12. Total acidity 50 . Benzidin +++ . Bile + .

October 21: Liver dulness the same. Splenic dulness 4.5 $\mathrm{cm}$. above costal margin. No hemorrhage. Vomited once yesterday. Patient is stuporous.

November 3: Redness and swelling of left eardrum; bulging; lanced; serum discharged. Pain subsided Novemher 2 and 3 . There was an elevation of temperature to 100 . The fever promptly subsided after the paracentesis was performed.

November 5: Free fluid demonstrable within abdominal cavity.

November 7: Three liters of serum removed by paracentesis of the aboiomen. The fluid was bile stained, and slightly cloudy; specific gravity 1.009 ; no coagulation on standing; henzidin reaction negative; bile ++ . The cells consisterl of 80 per cent. mononuclear and 20 per cent. polynuclear. There were only 126 cells per cubic millimeter.

November 11: Twenty-four hour urine was collected: Total nitrogen $9.072 \mathrm{gm}$.; ammonia $1.242 \mathrm{gm}$.; urea $6.272 \mathrm{gm}$; ammonia 13.6 per cent. of total nitrogen; urea 69.1 per cent. of total nitrogen.

November 15 to 17: All traces of bile have disappeared from the urine, nor did bile again appear in the urine.

November 22: There is a large amount of fluid in the abdomen. Jaundice has almost disappeared. The hepatic dulness has again returned, and the liver is now palpable at the costal border. The spleen is also palpable at the costal margin.

November 22: The patient had three violent epileptic convulsions during the day. From the following day the patient began to show signs of improvement.

December 8: Jaundice has returned. Liver palpable 1 inch below the costal margin. The spleen is at the costal margin.

December 19: Jaundice is no longer perceptible. The spleen is no longer palpable. No return of ascites.

Twenty-four hour urine on the day of discharge from hospital, December 19: amount 2,310 c.c.; ammonia 0.957 gm.; ammonia 5.04 per cent. of total nitrogen.

With the exception of two days when the patient had an otitis media there was no fever. There was never the slightest evidence of any renal disease, or cardiovascular impairment. The clinical picture was clearly that of an acute degencrative process in the liver. Although the large amount of ammonia eliminated in the urine at the time of the convulsions indicates an acidosis, there was no hyperpnea. The character of the ascitic fluid was decidedly that of a transudate.

\section{COMMENT}

It may justly be said that there is insufficient direct evidence of an elevation of portal pressure in the second case, but it was this experience which suggested one or two possible sources for the ascites, namely, either an elevation in portal pressure on account of an acute hepatitis, or morely a hydrops peritonei which happened to be the only collection of extravascular serum because of some modification of chemistry of the blood.
In the first case we wore dealing with an old interstitial hepatitis which renders the liver inelastic. An acute toxic hepatitis causing an increase in the parenchrmatous volume under such conditions would create a ligher pressure within the enclosure of Glisson's capsiile than would the same degree of parenchymatous ealargement when it occurs within the enclosure of a normal Glisson's capsule with normal and less resistant interstitial tissue.

The behavior of the audible hum and palpable thrill in the caput medusae in the first case is clear and direct evidence of elevation of portal pressure due to parenchymatous hepatitis in the presence of an old interstitial hepatitis.

In the second case, the evidence of the elevation of portal pressure due to parenchymatous hepatitis in a liver otherwise normal is inferential, but there are many experiences reported in perfusion of the liver which strongly suggest the possibility of sone chemical source for this increase in resistance to portal blood flow.

The first case may be explained on the purely physical basis of parenchymatous enlargement within an unyielding capsule and interstitial framework of the liver, but the second case demands another explanation.

Whatever the cause may be in the second case, the two experiences show how important it is to consider the part parenchymatous disease of the liver may play in the diagnosis and treatment of ascites from hepatic origin.

\section{PROTEOSE INTOXICATION}

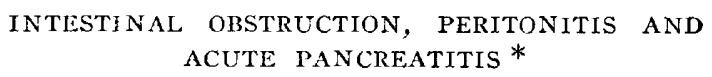

This communication deals with the etiology of the intoxication which develops in intestinal obstritction, general peritonitis and acute hemorrhagic pancrcatitis. It has been demonstrated ${ }^{1}$ that the intoxication of intestinal obstruction is due to a primary proteose which may be precipitated by five volumes of 95 per cent. alcohol or by half saturation with ammonium sulphate. It is comparatively easy to isolate the poison from closed loops of the intestine. This proteose is very toxic and $100 \mathrm{mg}$. may suffice to poison fatally a 15 pound dog.

Peritonitis and pancreatitis have some clinical features in common with acute intestinal obstruction. At times there may be some difficulties in differential diagnosis. We propose to show that the intoxication in these three conditions is due in large part to toxic proteoses. There may well be other substances concerned, but I believe that the proteose is the most important factor in the toxic reaction following peritonitis and pancreatitis.

It may be objected that when a toxic proteose is isolated from a closed loop of intestine this substance is not actually concerned in the intoxication. For example, it cannot be demonstrated in the blood. But when a toxic proteose is isolated from the exudate of a general peritonitis, no valid reason can be

* Read before the Association of American Physicians, Washington, D. C., May 9, 1916 .

* From the George Williams Hooper Foundation for Medical Research, University of California Medical School.

1. Whipple, Rodenbaugh and Kilgore: Jour. Exper. Med., 1916, xxiii, 123 . 